\title{
Treatment of heart failure with preserved ejection fraction
}

\author{
Alfredo Monteverde1, Valeria Carlino1, Giulia Ganci², Giuseppe Taormina1, \\ Mario Barbagallo1
}

\author{
1 Geriatrics Unit, Department of emerging diseases and of continuity of care, University Hospital \\ "Paolo Giaccone", Palermo
}

2 Agency for Health Care 5 "Friuli Occidentale", Cardiology Unit, Hospital of San Vito al Tagliamento (PN), Italy

\begin{abstract}
Heart failure, in its diverse forms based on the value of the ejection fraction, is associated to high mortality and the frequent need for hospitalization, with a consequent heavy burden on healthcare resources. For an appropriate treatment of heart failure with preserved ejection fraction (HFpEF), there are no specific drugs effective for this condition. Those indicated in HF with reduced EF (HFrEF) are of more benefit in that form of HF, according to the guidelines of the European Society of Cardiology of 2016: ACE-inhibitors, beta-blockers, anti-aldosterones are all indicated with a class of recommendation/level of evidence IA; therapy with loop diuretics is indicated in the case of clinical congestion (IB). Studies carried out with ACE-inhibitor (or angiotensin-receptor antagonist) or beta-blocker or anti-aldosterone to evaluate their efficacy also in HFpEF have substantially shown a benefit only on a reduction in the hospitalization rate. The same guidelines identify regular aerobic activity (IA) as more effective in the condition of HFpEF and indicate, appropriately, the need to treat comorbidities (IC) in the elderly (where they are more present and more numerous). They also recommend to avoid certain associations of drugs and, obviously, harmful (and contraindicated) medications that could worsen the clinical picture or might be potentially lethal.
\end{abstract}

Corresponding author: Dr. Alfredo Monteverde, Dipartimento delle patologie emergenti e della continuità assistenziale, U.0.C. di Geriatria, Policlinico, Via del Vespro 129, 90127, Palermo, Italy.

E-mail: alfredo.monteverde14@gmail.com

Key words: Ejection fraction; heart failure; comorbidity; guidelines; therapeutic strategies.

Received for publication: 24 April 2018

Accepted for publication: 14 May 2018

(C) Copyright A. Monteverde et al., 2018

Tipografia PI-ME Editrice, Italy

Monaldi Archives for Chest Disease 2018; 88:951

doi: 10.4081/monaldi.2018.951

This article is distributed under the terms of the Creative Commons Attribution Noncommercial License (by-nc 4.0) which permits any noncommercial use, distribution, and reproduction in any medium, provided the original author(s) and source are credited.
Heart failure (HF) is defined as being with preserved systolic function (HFpEF) if the ejection fraction (EF) is equal to or greater than $50 \%$ (in this case a diastolic dysfunction is present), or as HF with reduced systolic function (HFrEF) if the EF is $<40 \%$. Obviously, there is not always a clear-cut distinction between the two conditions and it is not infrequent to find decompensated patients with an intermediate (mid-range) EF between 40 and 49\% (HFmrEF) [1]. The first two conditions are differentiated in terms of their epidemiology, etiopathogenesis, clinical picture, and prognosis. While it is not within the scope of this article to investigate these differences, suffice to say that HFpEF represents approximately $50 \%$ of $\mathrm{HF}$ cases and it is more common in women and in the elderly. In the latter group, HFpEF is considered by some authors as an "exaggeration" of the aging process of the cardiovascular system [2]. Arterial hypertension is present in approximately $60 \%$ of patients with $\mathrm{HFpEF}$, and in the majority of patients comorbidities are also present (e.g., obesity, atrial fibrillation, renal failure, diabetes mellitus) which are more frequently observed and more numerous in the elderly. The pathogenesis of HFpEF is linked to microvascular alterations and an inflammatory state. The form of HFrEF, on the other hand, is prevalent in individuals affected by ischemic heart disease, with consequently the loss of myocytes underlying the pathogenesis of HF. The mid-range form (HFmrEF) is "closer" to the condition with preserved ejection fraction (HFpEF) in some respects (e.g. left atrial enlargement, elevated levels of natriuretic peptides, left ventricular hypertrophy and impaired diastolic release) [1]. Regarding the prognosis, HF is associated with high mortality and the need for frequent hospitalizations, with a very similar rate of hospitalization between the various forms, and hence the disease represents a heavy burden on healthcare resources. In patients with HFpEF, the prognosis is clearly worse if there is concomitant arterial hypertension. If, in addition, blood pressure is low at the time of admission, these patients also have a higher risk of mortality.

In dealing here with the topic of appropriate therapy in heart failure, while we are not able (nor do we pretend) to give an exhaustive account, we cannot not refer to what the guidelines stipulate regarding the drugs that are indicated or contraindicated. HFrEF therapy is based on the use of angiotensin-converting-enzyme (ACE) inhibitors, betablockers, and mineralocorticoid receptor antagonists: these, according to the 2016 guidelines of the European Society of Cardiology (ESC), are all indicated with a recommendation class/level of evidence IA [1], while angiotensin II receptor blockers (ARBs) have a slightly lower level of indication: IB. Several studies have been conducted to verify the efficacy of these pharmacological treatments, also in HF patients with diastolic dysfunction (HFpEF) but without using $\mathrm{EF} \geq 50 \%$ as the cut-off defining HFpEF. In fact, the CHARM-Preserved trial, dating back to 2003 , enrolled patients with an $\mathrm{EF} \geq 40 \%$, and showed a positive ef- 
fect of candesartan only in reducing hospitalizations [3]; the PEP-CHF study, carried out in patients aged $>70$ years and using the same $40 \%$ cut-off for EF, showed no benefit of perindopril on symptoms or on reducing the need for hospitalization [4]; in the I-Preserved study, carried out in patients with an $\mathrm{EF}$ limit of $45 \%$ (once again, a value lower than the $50 \%$ cut-off), no significant benefit of irbesartan on survival or hospitalization was found [5]; the TOPCAT trial, which evaluated patients with an $\mathrm{EF} \geq 45 \%$, reported a benefit of spironolactone only on hospital admissions [6]. Although the use of such drugs (ACE-inhibitors, beta-blockers, anti-aldosterones, and ARBs) is more effective in the HFrEF condition (i.e., with $\mathrm{EF}<40 \%$ ), they are also used in the case of HFpEF given their recognized ability to reestablish an equilibrium in the neuro-endocrine modifications underlying the HF pathophysiology and their role - not to be overlooked - in the control of systemic blood pressure and heart rate and in slowing down, at least, the clinical decline of HF and improving patients' quality of life. More recently, a new drug of the angiotensin receptor-neprilysin inhibitor (ARNi) class, sacubitril/valsartan, used in HF with reduced systolic function (HFrEF) was found superior to enalapril in reducing the risk of mortality or hospitalization in heart failure [7]. This new compound is undergoing investigation to verify eventual benefits also in HFpEF, and ranolazine is also being evaluated in diastolic dysfunction. We are waiting for the results of these trials.

It should be pointed out here that diastolic dysfunction (which can also be asymptomatic) is not in itself an equivalent of HFpEF which, to be defined as such, requires signs and symptoms indicative of altered hemodynamic balance at clinical presentation. It is commonly retained, therefore, that at present there are no drugs available with specific efficacy for the treatment of diastolic dysfunction and/or HFpEF and that there is no drug therapy that can reduce the mortality, as further confirmed by a meta-analysis published in 2011 [8]. The ESC 2016 guidelines indicate for this condition, when a pattern of congestion is present, diuretics - loop diuretics are preferable (IB) - but, above all, in a treatment strategy not based only on drugs, regular aerobic physical activity (IA). In addition, the need to treat the comorbidities (IC) is appropriately indicated. One should also emphasize the need to promptly and appropriately address factors that could precipitate cardiac decompensation (e.g., anemia, sepsis, chronic obstructive pulmonary disease) and, at the same time, avoid toxic substances (e.g., alcohol and drugs) and all harmful and dangerous medications such as glitazones (IIIA according to the ESC 2016 guidelines) or COX-2 cyclooxygenase inhibitors (IIIB) which increase the risk of clinical decline and of hospitalization, as well as diltiazem and verapamil (III C) for their negative inotropic effect and, in the case of arrhythmias, dronedarone and the class 1 antiarrhythmics (both IIIA), due to their proarrhythmic and potentially lethal effect. Also contraindicated is the association of an- giotensin (or renin) receptor inhibitors with ACE-inhibitors and mineralocorticoid receptor antagonists (III C) due to the risk of renal dysfunction (or its worsening) and hyperkalemia [1].

An early recognition of the cause (or causes) of HFpEF is also fundamental in order to increase the probability of an improvement of the condition: one thinks of particular forms of HFpEf, such as amyloidosis, Fabry's disease, familial cardiomyopathies. Lastly, within the framework of a global treatment strategy (not only pharmacological), we stress the importance of a multidisciplinary approach, including the participation of the family doctor, nurse, physiotherapist, psychologist, social worker, and possibly surgeon in the patient's management, besides the cardiologist who nevertheless maintains the primary role. And, finally, one should not forget the importance of periodically reviewing the therapy and dosage of drugs, particularly in elderly patients, after a careful clinical monitoring in order to reduce the number of drugs prescribed (where possible) and suspend those that are ineffective and/or have no positive benefit on the patient's quality of life [1].

\section{References}

1. Ponikowski P, Voors AA, Anker SD, et al. 2016 ESC Guidelines for the diagnosis and treatment of acute and chronic heart failure. Eur Heart J 2016;37:2129-200.

2. Oxenham H, Sharpe N. Cardiovascular aging and heart failure. Eur J Heart Fail 2003;5:427-34.

3. Yusuf S, Pfeffer MA, Swedberg K, et al. Effects of candesartan in patients with chronic heart failure and preserved left ventricular ejection fraction: the CHARM-Preserved Trial. Lancet 2003;362:777-81.

4. Cleland JGF, Tendera M, Adamus J, et al. The perindopril in elderly people with chronic heart failure (PEP-CHF study). Eur Heart J 2006;27:2338-45.

5. Massie BM, Carson PE, McMurray JJV, et al. Irbesartan in patients with heart failure and preserved ejection fraction. N Engl J Med 2008;359:2456-67.

6. Pitt B, Pfeffer MA, Assmann SF, et al. Treatment of preserved cardiac function heart failure with an aldosterone antagonist (TOPCAT). N Engl J Med 2014;370:1383-92.

7. McMurray JV, Packer M, Desai AS, et al. Angiotensin-neprilysin inhibition versus enalapril in heart failure. $\mathrm{N}$ Engl $\mathrm{J}$ Med 2014;371:993-1004.

8. Holland DJ, Kumbhani DJ, Ahmed SH, Marwick TH. Effects of treatment on exercise tolerance, cardiac function and mortality in heart failure with preserved ejection fraction. A meta-analisys. J Am Coll Cardiol 2011;57:1676-86. 